\title{
Parental awareness on anti-epileptic treatment
}

\author{
J Kudalugodaarachchi ${ }^{1}$, Y S de Alwis ${ }^{2}$, K K Samarage ${ }^{3}$ \\ Sri Lanka Journal of Child Health, 2003; 32: 69-70
}

(Key words: Parental awareness, anti-epileptic therapy, epilepsy, children)

\begin{abstract}
Objective To assess awareness on anti-epileptic therapy among parents of epileptic children.
\end{abstract}

Setting Epilepsy clinic, University Paediatric Unit, Colombo South Teaching Hospital.

Study population All patients registered in clinic, followed up for at least 3 months.

Method An interviewer administered questionnaire was used covering 16 aspects of anti-epileptic therapy. Responses for each aspect were graded as excellent $(70 \%$ or $>)$, good $(50-69 \%)$ or poor $(<50 \%)$.

Results Parents of 58 children were interviewed. $100 \%$ parents had been advised on general aspects of epilepsy and $92 \%$ on principles of anti-epileptic drug (AED) therapy. In 93\% this knowledge had been imparted to parents by a medical officer. In $96 \%$ this advice had been given at onset of treatment and in $64 \%$ it had been reinforced subsequently. A 'good' or 'excellent' response was obtained in 11 aspects of AED therapy whilst a 'poor' response was obtained in the remaining 5 aspects.

Conclusions Parental awareness of certain aspects of AED therapy needs to be improved.

\section{Introduction}

Epilepsy is the most common neurologic disease in children and adolescents ${ }^{1}$. The rate of new diagnoses (i.e. the incidence rate) of childhood epilepsy ranged from $20 / 100,000$ to $375 / 100,000$ dependent on age group, gender and country ${ }^{1}$. Epilepsy in childhood generally has a good prognosis ${ }^{2}$. Most types respond well to conventional anti-epileptic drugs (AEDs) which have to be used over a prolonged period. Continuation of AEDs without interruption is vital.

${ }^{1}$ Registrar, ${ }^{2}$ House Officer, Professorial Unit, Colombo South Teaching Hospital, ${ }^{3}$ Senior Lecturer in Paediatrics, Faculty of Medical Sciences, University of Sri Jayawardenepura.

(Received on 21 July 2003)
Adjustment of the therapeutic regimen may be required depending on the clinical response, doses being altered according to the weight of the patient.

Parental understanding of the disease and its treatment is an important general principle accepted as desirable in management of any chronic illness ${ }^{3}$. Improvement in epileptic care is based on a shared understanding between the doctor, patient and parent ${ }^{4,5}$.

\section{Objective}

This study was done to assess the awareness on antiepileptic therapy among parents of epileptic children.

\section{Method}

The study was carried out at the epilepsy clinic, University Paediatric Unit, Colombo South Teaching Hospital. The study group included all patients who had been registered and followed up in the clinic for a minimum period of 3 months.

An interviewer administered questionnaire was used. This had both open-ended and close-ended responses. There were 30 questions, each of which constituted an audit item. These items were formulated to cover 16 aspects pertaining to principles of anti-epileptic therapy. The number of correct responses marked for each aspect was graded as follows: $70 \%$ or more as 'excellent', 50-69\% as 'good' and less than 50\% as 'poor' level of understanding on principles of AED therapy.

\section{Results}

Parents of 58 children were interviewed. Mean duration of follow up was 1 year and 8 months. Age distribution of epileptic children ranged from 1-14 years with a mean age of 5.8 years. Parental level of education was as follows: $3.4 \%$ had not received any form of education, $5.2 \%$ had an educational level less than Grade 5, 81\% had a level of education ranging from Grades 5-10 and 10,3\% had an educational level more than Grade 10. 
$100 \%$ parents had been advised on general aspects of epilepsy at some point in their follow up. 92\% had been advised on principles of AED therapy. This knowledge had been imparted to parents by a medical officer in $93 \%$ while the rest had been educated by nursing officers and medical students. In $96 \%$ this advice had been given at onset of treatment and it had been reinforced further in $64 \%$ either at the clinic or on a subsequent hospital admission.

An 'excellent' level of knowledge $(70 \%$ or $>)$ was shown in 6 aspects of AED therapy. This included knowledge on the required duration of therapy, importance of regular reviews during this period, importance of maintaining regular drug supplies at all times, need for continuation of treatment during inter-current infection, ability to identify tablet by its appearance and importance of dispensing drugs to the child by a responsible person.

A score of 50-69\%, denoting a 'good' level of knowledge, was shown in 5 aspects of AED therapy. These included ability to give generic name of the currently prescribed drug/drugs, knowing prescribed dosage in milligrams, knowing approximate cost of drugs prescribed for their child, knowing the importance of weight in calculating drug doses and questioning doctors and other medical staff regarding treatment of epilepsy indicating interest and curiosity on the subject.

A 'poor' level of knowledge $(<50 \%)$ was shown regarding 5 aspects of AED therapy. These included tablet strength of prescribed drug, drug interactions, common side effects associated with anticonvulsants, some common brand names of the regular antiepileptics and the various sources of information regarding AEDs.

\section{Discussion}

An epilepsy clinic is an important patient care facility in adult medicine as well as in paediatrics. It gives enough time and opportunity to review these patients more leisurely in a relaxed atmosphere instead of in the usual overcrowded general clinic which offers short consultations.

Knowledge of epilepsy patients about their disorder, treatment and consequences is often poor ${ }^{6,7}$. A controlled, randomized study demonstrated the efficacy of an educational treatment program for adult patients with epilepsy ${ }^{5}$. A randomized field trial has been done in children with chronic illnesses (asthma and epilepsy) ${ }^{8}$. Parents play the most important role in ensuring satisfactory compliance to
AEDs. Therefore, advising the parent is an essential component of good epilepsy care. Success of treatment depends on how well the parent understands the principles of AED therapy. As far as we are aware, no previous studies have been done to assess parental awareness on AEDs in paediatrics.

A clinical audit is useful to identify drawbacks and weaknesses in the care offered to our patients. In this study there were many identified areas which had been covered inadequately. These included knowledge on side effects and interactions associated with AEDs, tablet strength etc. These aspects are often not communicated to parents in our general paediatric practice. This study highlights the need for spending more time with the parents and further improvement of deficiencies identified.

\section{Conclusion}

Parental awareness of certain aspects of AED therapy, such as side effects and drug interactions, needs to be improved.

\section{References}

1. Freitag C M, May $\mathrm{T}$ W, Pfafflin M, Konig S, Rating D. Incidence of epilepsies and epileptic syndromes in children and adolescents: A population-based prospective study in Germany. Epilepsia 2001; 42(8): 979-85.

2. Camfield P, Camfield C. Epileptic syndromes in childhood: clinical features, outcomes and treatment. Epilepsia 2002; 43(s3): 27-32.

3. Kalra V. Management of childhood epilepsy. Indian Journal of Paediatrics 2000; 67(1 Suppl): S12-21.

4. Nordli D R. Special needs of the adolescent with epilepsy. Epilepsia 2001; 42(s8): 10-17.

5. May $\mathrm{T}$ W, Pfafflin $M$. The efficacy of an educational treatment program for patients with epilepsy (MOSES): Results of a controlled, randomized study. Epilepsia 2002; 43(5): 539-49.

6. Long L, Reeves AL, Moore J L. An assessment of patients' knowledge of their disorder. Epilepsia 2000; 41: 727-31.

7. Dawkins J L, Crawford P M, Stammers T G. Epilepsy; a general practice study of knowledge and attitudes among sufferers and non-sufferers. $\mathrm{Br}$ J Gen Pract 1993; 43: 453-7. 
